\title{
Sistema de Recomendação para apoio à colaboração em
}

ambientes baseados em conhecimento

Stanley Loh ${ }^{1,2}$, Daniel Lichtnow ${ }^{1}$, Ramiro Saldaña ${ }^{1}$, Rodrigo Branco Kickhöfel ${ }^{1}$ Stanley Limões', Thyago Borges ${ }^{1}$, Tiago Primo', Gustavo Piltcher', Luiz Carlos Ribeiro Junior ${ }^{1}$, Cleber Gouvêa

'Universidade Católica de Pelotas (UCPEL) - Grupo de Pesquisa em Sistemas de Informação R. Félix da Cunha, 412, Pelotas, RS - CEP 96010-000

2Universidade Luterana do Brasil (ULBRA) - Faculdade de Informática R. Miguel Tostes, 101, Canoas, RS - CEP 92420-280

\{lichtnow, rsaldana, rodrigok, thyago, lcr\}@ucpel.tche.br liotra com.br, gsimoes@vetorial net, tiagoprimo@brturbo.com, gustavopil@yahoo.com.br, cleberg@email.com.br

Abstract. This paper presents a recommender system to support collaboration among people. The system provides a private chat room, where people can exchange textual messages. Analyzing the messages, the system discovers the themes discussed and then it can help people by recommending complementary knowledge sources. Furthermore, chat sessions are stored and can be retrieved later, so that people can review decisions processes or learn from past discussions. Statistical analyses of the sessions can reveal important knowledge about the discussions.

Resumo. Este artigo apresenta um sistema de recomendacão para apoio a colaboração através de pessoas. O sistema dispõe de uma sala de chat privada, onde pode-se trocar mensagens textuais. Analisando as mensagens, o sistema descobre os temas discutidos e então este poderá ajudar outras pessoas, recomendando fontes complementares de conhecimento. Além disso, as sessões de chat são armazenadas, podendo ser recuperadas futuramente, então, essas pessoas poderão rever processos decisórios ou aprender com as discussões passadas. Análises estatisticas das sessões podem revelar importantes conhecimentos sobre as discussões.

Introducão

sucesso

pos de uma organizacão está relacionado em grande parte ao conhecimento que possui. O conhecimento de uma organização é formado a partir do conhecimento Seus membros e pode aumentar à medida que este é armazenado e compartilhado.

e o conceito de organizaçoes que aprendem (learning organization), conforme $\mathrm{GE}(2001)$

Segundo NONAKA e TAKEUCHI (1995) a maior parte do conhecimento ganizacional vem da interação entre pessoas. Pessoas tendem a reusar soluções de pessoas para ganhar produtividade.

Nesse contexto de valorização do conhecimento como um recurso corporativo, chamada Gestão do Conhecimento (Knowledge Management - KM). A Gestão 
do Conhecimento tem seu foco voltado para questões relacionadas a como organizações podem tirar maior proveito do conhecimento existente dentro de facilitando a distribuição deste conhecimento entre seus membros, encorajando o rela das soluções adotadas para resolução de problemas, evitando a perda do conhecimisto de seus especialistas quando estes deixam a organização (DAVENPORT \& PRUZ 1997).

Quando pessoas se comunicam para trocar informação ou para adquiri conhecimento a fim de realizar um trabalho ou ajudar outra pessoa, o processo chamado Colaboração. A colaboração pode ser realizada através de interações síncro (por exemplo, um chat), assíncronas (como fóruns), contato direto (pessoas falando) contato indireto (quando pessoas armazenam conhecimento e outras podem recuperá-lo a qualquer momento)

A colaboração é uma das mais importantes tarefas para a inovação e vantagem competitiva (SENGE, 2001). Ela também é importante no armazenamento do conhecimento para uso posterior. Se o conhecimento não é adequadamente registrado organizado e recuperado, a conseqüência é o re-trabalho, baixa produtividade e perda de oportunidades.

Em uma equipe de trabalho, cada indivíduo carrega consigo uma série de conhecimentos tácitos que, se disseminados para os outros membros, podem contribuir de forma expressiva para uma melhor eficácia na execução dos processos, resultando assim em sucesso organizacional. A capacidade de mapear o conhecimento tácito de cada membro da equipe gera valor para a organização, já que assim, pode-se evitar a necessidade de contratar pessoas ou serviços buscando tal conhecimento, pois o mesmo foi identificado em alguém que faz parte do time.

Segundo DAVENPORT e PRUZAC (1997), gigantes como HP e IBM não possuem a menor idéia de como gerenciar o valor agregado das informações e conhecimentos de suas empresas. Sabendo disso, a proposta de uma ferramenta que auxilie na resolução destes problemas, sem duvida, seria de grande valia para muitas organizações de médio e grande porte.

Indo mais além, um desafio para Gestão do Conhecimento é usar o conhecimento de uma forma pró-ativa, ou seja, se antecipando às ações do usuário. Uma tecnologia que pode auxiliar nessa tarefa são os sistemas de recomendação. O objetivo principal desses sistemas é localizar fontes de informação relacionadas ao interesse ou necessidade do usuário (MONTANER et al., 2002), ou seja, eles fornecem informação sem que 0 usuário precise realizar uma busca.

Visto a grande necessidade que as empresas têm de gerenciar sua memória organizacional e aumentar a produtividade através de um melhor aproveitamento de seus recursos, este trabalho apresenta um sistema de recomendação que apóia a colaboração entre pessoas. O sistema constitui uma Biblioteca Digital que armazena os recursos da empresa, e um chat web onde os usuários realizam interações síncronas e recebem recomendações conforme os assuntos abordados. Essas recomendações podem ser de itens da Biblioteca Digital, de discussões anteriores (sessões de chat já realizadas) ${ }^{e}$ usuários com certo grau de conhecimento no assunto abordado.

Na seção 2 deste artigo, são aprofundados alguns aspectos relacionados a0s Sistemas de Recomendação e sistemas existentes. A seção 3 apresentada a arquitetura do sistema proposto. Por fim, na seção 4 , as conclusões discutem contribuições e limitậo es da proposta, bem como informações referentes a daçeres. São listados, também nas implementado, saios encontrados na implementação e implantação do protótipo.

conclusões, desafios encelatos

2. Sistemas de Recomendação éc é Um grande problema na maioria dó -ativa. Dessa forma o usuário precisa realizar uma não fazem isso de conhecimento para encontrar o que interessa.

Por exemplo, as Bibliotecas Digitais são importantes no processo de gestão do , pessoas precisam procurar em grandes volumes de itens. conhecimento, entretante as 1989) compara as bibliotecas atuais com as do futuro. As Feigenbaum (apud DAVIES, atuais consistem de un a descobrir novos fituro serão coleções de documentos ativos, ajuda futuro simentos, fornecendo associações previamente desconhecidas, analogias e novo conhecimentos, for as pessoas tenham que indicar claramente suas necessidades de conceitos seração.

Nesse contexto os sistemas de recomendação são uma tecnologia emergente. Seu 列

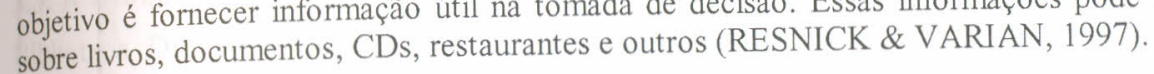

TERVEEN e HILL (2001) discutem vários sistemas de recomendação. Por exemplo, o sistema PHOAKS extrai páginas $W e b$ que foram mencionadas em mensagen do newsgroup Usenet para futuramente permitir a recuperação destas indicações. Já o do new sistema proposto por Donath et al. (apud TERVEEN \& HILL, 2001) analisa discussões do Usenet e de chats, atribuindo-lhes propriedades (por exemplo, número de participações). Isto permite recuperar discussões com certas propriedades. Estas ferramen a discussão. Neste caso, a função é apenas a de registrar as mensagens para permitir a posterior recuperação de informações.

Já o sistema Tapestry permite que as pessoas registrem suas avaliações sobre certas mensagens postadas em uma discussão. Entretanto, neste caso, os processos de armazenar e recuperar conhecimento acontecem por iniciativa do usuário.

Um exemplo de sistema pró-ativo (sistema que realiza alguma tarefa sem que o usuário precise solicitá-la) voltado para apoio a interações é apresentado em KOMOSINSKI et al. (2000). Este sistema identifica termos presentes nas mensagens trocadas entre os participantes de um chat e apresenta a definição de tais termos durante a interação. Entretanto, esta ferramenta não oferece nenhum tipo de recomendação, mas somente explicações dos termos usados.

\section{Sistema de Recomendacão para apoio a Colaboração}

Para auxiliar na colaboração entre pessoas e contribuir com a Gestão de Conhecimento nas organizações foi descnvolvido um sistema chamado de SisRecCol.

O SisRecCol (Sistema de Recomendação para apoio a Colaboração) consiste em um chat Web ond assunto identificado nas mensagens. Essa identificação é realizada através de técnicas de text mining apoiadas em uma ontologia desenvolvida para o sistema. As recomendações 
são de documentos eletrônicos e links para páginas da Web (armazenados Biblioteca Digital privada do sistema), de autoridades nos assuntos (identificados nu próprio sistema) e de discussões anteriores sobre o mesmo tema.

O objetivo do SisRecCol é apoiar a troca de conhecimento entre os usuá permitir o armazenamento e recuperação de conhecimento explicito e também táci e através do armazenamento das sessões de chat. O sistema pode ser adaptado para se utilizado em diferentes áreas, bastando incluir uma nova ontologia, referente ao no domínio. O protótipo do sistema está disponível em http://gpsi.ucpel.tche.br. Na figuvo é possível ver a tela do chat com um exemplo de discussão e a lista de recomendacõ referentes ao assunto abordado.

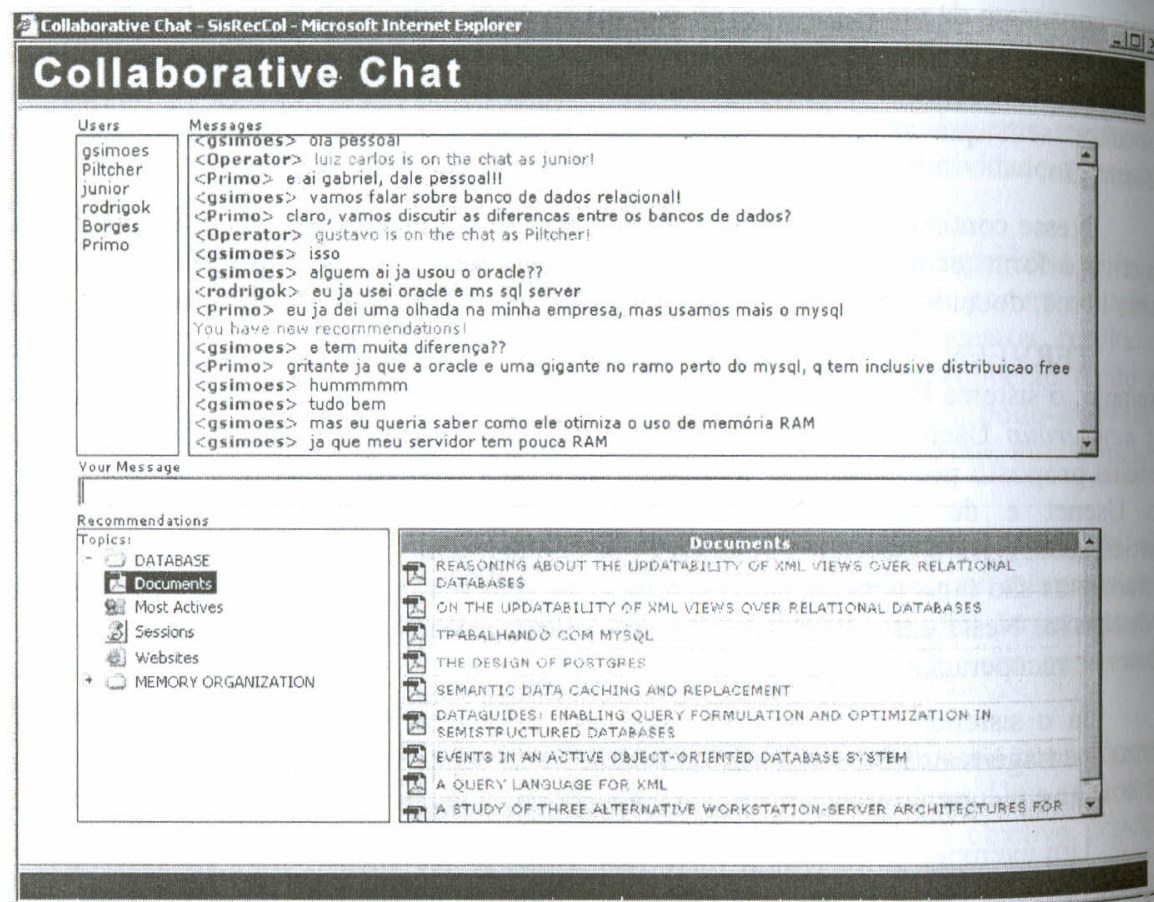

Figura 1. A interface do chat, mostrando recomendações

\subsection{A Ontologia}

A base do sistema é uma ontologia de domínio utilizada para classificar itens na biblioteca digital, para identificar temas nas mensagens e para traçar o perfil dos usuarios.

Uma ontologia é uma definição formal e explícita de conceitos (classes ou categorias) e seus atributos e relações (NOY \& MCGUINNES, 2002). Uma ontolog do domínio - domain ontology é uma descrição de "coisas" que existem ou poder existir em um domínio (SOWA, 1997).
No SisRecCol a ontologia é apresentada como uma hierarquia de conceitos e Ela deve ser criada para o domínio da conceito contem un seja, deve conter os conceitos e termos da área que será cada aplicação de aplicara.

\subsection{Módulo de Text Mining}

ídulo de Text Mining é responsável pela identificação dos assuntos das mensagen Fle realiza o batimento entre as palavras da mensagem com os termos presentes do chat. Ele realiza ó de métodos probabilísticos, identifica o assunto referente. Essa na ontologia e, atraves de metodos prom, analisando um identificação não ocorre apenas importância contexto (se varias mensagens tratam do contexto (se veterminado parâmetro, a probabilidade desse assunto ser o tôn acima de um deterna esse então, identificado como assunto principal desse conjunto de discussão é

Mining também é utilizado para a indexação dos documentos

O módulo de Text Minih presentes na biblioteca digital.

\subsection{A Biblioteca Digital}

Biblioteca Digital é uma coleção de recursos digitais, organizados sob certa lógica, Biblioteca Dis recuperação sobre uma rede de computadores (KOCHTANEK et al., 2001).

A Biblioteca Digital do SisRecCol contém documentos eletrônicos e links para páginas Web, no momento. Este conteúdo é previamente indexado com ferramentas de paginas Web, no mom mesmos métodos software que realizam o processo das mensagens do chat. O processo de indexação determina o grau de relacionamento dos documentos, (artigo ou site) aos conceitos existentes na ontologia.

A inclusão dos itens na Biblioteca Digital pode ser feita pelos usuários, num processo independente da sessão do chat.

\subsection{A Base de Perfis}

A base de perfis contém a identificação dos usuários e suas áreas de conhecimento. A base se assemelha aos chamados Mapas do Conhecimento ou Páginas Amarelas (Yellow Pages), que servem para indicar que pessoas possuem determinados conhecimentos. Mapa do Conhecimento é, portanto, um guia e não um repositório de conhecimento (STEWART, 1998)

A indicação dos conhecimentos que as pessoas possuem é feita através de uma relação de conceitos (dentre os presentes na ontologia) e um grau associado a cada conceito. Este grau determina puanto uma pessoa está relacionada à um certo assunto ou tema do domínio. O grau não é absoluto, mas relativo às demais pessoas do grupo. Este valor pode ser usado para indicar o grau de competência da pessoa na área ou para disenciar se uma pessoa está mais ativa do que as outras em determinada área. Alem disso é dinâmico, sendo modelado conforme o usuário utiliza o sistema. 


\subsection{Módulo de Recomendação}

A partir do assunto identificado nas mensagens do chat, o módulo de recomendaçã ativamente sugere a cada usuário uma lista de itens relevantes vindos da Bibâa $p$ p Digital (documentos, páginas da Web), sessões anteriores e da base de perfis (ubliote com conhecimento no assunto).

Essa recomendação é apresentada em uma área separada, não interfer. discussão, e permite que o usuário acesse o conteúdo, por exemplo, abrir um docundo para leitura,

Os itens que serão recomendados são definidos conforme o perfil de usuário. Se um usuário já leu determinado documento ele não será recomendad também um documento básico não é recomendado para um usuário avançado.

Além das recomendações geradas durante as sessões de chat, o sistem apresenta métodos de recomendação off-line que estão disponíveis a momento. Esses métodos utilizam informações como "os civeis ao usuário a qualque usuários mais ativos" e "os documentos lidos por todos os usuários", gentos lidos pelos por exemplo, dos documentos mais lidos pelos nos últimos 30 dias ou nos útimos 30 dias ou os documentos mais lidos no assunto de maior interessem usuário que recebe essa recomendação. O interesse do usuário é definido através de seu perfil, conforme citado anteriormente.

\subsection{Módulo de Estatísticas}

O sistema possui um módulo de estatísticas que, conforme exibido em exemplo resumido na figura 2 , consiste de um conjunto de ferramentas utilizadas na análise des discumido ocorridas durante as sessões de chat.

As estatísticas permitem verificar a interação de cada usuário durante as sessões, descobrindo seu tipo de interesse. Além disso, é possível verificar quem são os usuários mais ativos, os menos ativos (apenas assistem a discussão), se os usuários falam mais sobre assuntos importantes (presentes na ontologia), entre outros.

A análise da ordem em que os conceitos vão sendo identificados permite descobrir como a discussão se desenrolou, ou seja, a rota da discussão pelos conceitos, permitindo também verificar os desvios do tema central.

Este tipo de análise também permite inferir a expertise do grupo como um todo, ou seja, que áreas são de maior conhecimento do grupo e que sub-áreas de um assunto não foram discutidas.
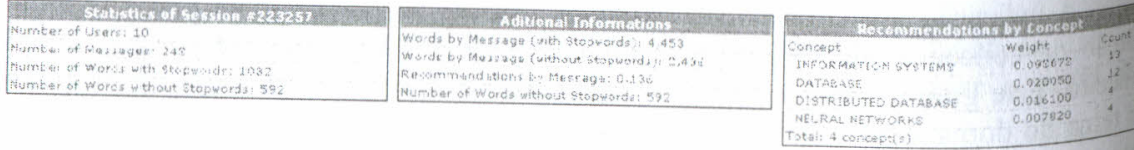

Figura 2. Algumas métricas do módulo de estatísticas
4. Conclusões Este trabalho aprocesso de transferência do conhecimento entre os membros de uma sistema auxilia no prando o aprendizado organizacional. Além disso, ele dá suporte ao ganização, facilitando armazenamentórios, quanto pela base de sessões de chat que torna explícito o

próprios conhecimento tácito dos usuários.

Atualmente o SisRecCol possui apenas uma ontologia de domínio voltada para a Ciam do Computação. Dessa forma foram realizados experimentos com grupos área de Cien Universidades. Com os testes pôde-se constatar que pessoas conseguem de pesquisa enthor o conhecimento com a ajuda das recomendações, pois o sistema comparticis a documentos e sites existem sobre o assunto. O compartilhamento mesmo lesore indiretamente, pois os usuários podem armazenar na Biblioteca Digital também ocorre links para sites, os quais poderão ser recomendados para outras pessoas. Além disso, o conhecimento presente nas discussões fica armazenado e pode ser recuperado através de consultas (ex: que discussões anteriores trataram do tema X) ou poderão ser recomendadas nas próprias sessões de chat.

método utilizado para identificar os assuntos das mensagens do chat foi avaliado formalmente, tendo-se medido que a precisão na identificação de conceitos ultrapassou os $85 \%$ de acerto.

Trabalhando as informações adquiridas com as sessões de chat, o sistema proposto permite, além de aumentar a base de conhecimentos da organização, a criação de um mapa do conhecimento organizacional, desde que seus membros utilizem a ferramenta. Este mapa possibilita a criação de Yellow-Pages, o que agilizará os processos no momento de buscar membros qualificados, ou até mesmo de delegar funções.

Embora hajam benefícios previstos, é preciso considerar que a aplicação do sistema poderá encontrar algumas barreiras. Uma delas reside no fato da base de conhecimento estar inicialmente com um volume pequeno, o que irá fazer com que não sejam feitas recomendações ou que estas sejam irrelevantes no início da aplicação da ferramenta. Neste sentido, é necessário incentivar o uso do sistema e o armazenamento prévio de documentos e sites relacionados aos assuntos de interesse da organização, até que 0 mesmo atinja um nível satisfatório de relevância.

Um desafio gerado pelo uso do sistema proposto é a necessidade de modificar o comportamento das pessoas envolvidas. Isso se dá já que as pessoas precisam entender que, se não utilizarem o sistema, o mesmo deixará de trazer qualquer beneficio a elas próprias e a organização.

Uma outra dificuldade é a criação da ontologia. Atualmente parte do trabalho é selecion pois requer que um especialista informe os conceitos da área de aplicação e configure manualmente documentos referentes a cada conceito para realizar a alternação das palavras na ontologia (através de ferramentas do próprio sistema). Uma automativa que já está em estudo seria a criação de uma ferramenta que identifique os documente os assuntos e ainda tenha capacidade de buscar na Web, por exemplo, 
Por permitir a recuperação de sessões anteriores de chat, o sistema prop colabora de forma ativa na disseminação de informações. Além disso, contribui pro crescimento da base de conhecimentos da organização, já que, analisando as men das sessões, é possível identificar os assuntos tratados, o que permite a identifica expertises dos usuários.

\section{Agradecimentos}

O presente trabalho foi realizado com o apoio do CNPq, uma entidade Governo Brasileiro voltada ao desenvolvimento científico e tecnológico.

\section{Referências}

Davenport, T. H. \& Pruzac, L. (1997) "Working knowledge - How organizatio manage what they know", Harvard Business School Press, Harvard.

Davies, R. (1989) The creation of new knowledge by information retrieval classification, Journal of Documentation 45 p.273-301.

Kochtanek, T. R. et al. (2001) "A digital library resource Web site: Project DL", Onl Information Review, v.25, p.29-40.

Komosinski, L. J. et al. (2000) Uso de Agentes para Apoio à Mediação de Diálo Entre Estudantes via Internet. In: Congresso Iberoamericano de Informática Educação (RIBIE 2000), V, Dez, 2000. Proceedings... Viña del Mar (publicado e CD).

Montaner, M. et al. (2002) Improving case representation and case base maintenance recommender agents, in: Proc. 6th European Conference on Case Based Reasoning.

Nonaka, 1. \& Takeuchi, T. (1995) "The knowledge-creating company: how japane companies create the dynamics of innovation", Oxford University Press, Cambridge.

Noy N. F. \& McGuiness, D. L. (2002) "Ontology Development 101: a guide to creat your first ontology". Disponível em http://protege.stanford.edu/publications/.

Resnick, P. \& Varian, H. (1997) "Recommender Systems" Communications of the AC v.40 p.56-58

Senge, Peter M. (2001) A quinta disciplina: arte e prática da organização que aprende. ed. Best Seller, 444p.

Sowa, J. F. (1997) "Building, sharing, and merging ontologies", AAAI Press / MI press, p3-41.

Stewart, T. A. (1998) Intellectual capital: the new wealth of organizations, Banta Books, 320p.

Terveen, L. \& Hill, W. (2001) "Beyond recommender systems: helping people help ead other", in: J. CARROLL, ed, Human computer interaction in the new millennium Addison-Wesley. 